\title{
Ultrasound-Guided Fine Needle Aspiration (USFNA) of Thyroid Nodules; Does Aspiration Site Matter?
}

\author{
Umit Aksoy Ozcan ${ }^{1, *}$; Safak Atahan ${ }^{2}$ \\ ${ }^{1}$ Department of Radiology, School of Medicine, Acibadem University, Istanbul, Turkey \\ ${ }^{2}$ Patonet Cytopathology Laboratory, Bursa, Turkey \\ ${ }^{*}$ Corresponding author: Umit Aksoy Ozcan, Department of Radiology, School of Medicine, Acibadem University, Istanbul, Turkey. Tel: +90-2165714426, Fax: +90-2165332352, E-mail: \\ umitozcan@gmail.com
}

Received: September 24, 2012; Revised: March 1, 2013; Accepted: September 27, 2013

Background: The goal of ultrasound guided fine-needle aspiration (USFNA) is to obtain most cellular specimen that represents the nodule. However, there is substantial variability in specimen cellularity depending on the obtaining techniques. While performing USFNA, it is not clear whether the needle tip should be placed at hypovascular or hypervascular site of the nodule to obtain more cells for cytological analysis.

Objectives: The aim of the study was to assess whether USFNA of the hypovascular or hypervascular site of a thyroid nodule would reveal more cells for cytological analysis.

Patients and Methods: Twenty-three consecutive patients with solid thyroid nodules larger than $2 \mathrm{~cm}$ were aspirated under color Doppler sonography guidance. First pass was from the hypovascular site and the second pass from the hypervascular site. The aspirates were scored from 1-3 by cytologist according to number of cell groups.

Results: When only hypervascular site was used, adequate sampling was limited to $65 \%$ of the cases. When both sites were evaluated together, overall adequate sampling was 91\%. Adding a sample from the hypervascular site to hypovascular aspirate increased the adequate sampling by $8 \%$. The highest pathological score was achieved when both hypo- and hypervascular site aspirates were evaluated together. Conclusions: The aspirates from the hypovascular and hypervascular sites of solid nodules are complementary and should be performed consecutively.

Keywords: Thyroid; Ultrasonography; biopsy; Fine Needle Aspiration; Doppler; Color

\section{Background}

Ultrasound guided fine-needle aspiration (USFNA) is accepted as the least invasive and most accurate method of identifying the nature of thyroid nodules $(1,2)$. During the procedure, the goal is to obtain the most cellular specimen that represents the target nodule. Published literature revealed substantial variability in USFNA specimen cellularity, ranged from $66.4 \%$ to $96.6 \%$ depending on different specimen obtaining techniques (2, 3). During aspiration, color Doppler sonography (CDS) is usually used to identify the perinodular and intranodular vessels to provide a safe access site and reduce the amount of blood in the aspirate as possible (1). The guidelines for aspiration of thyroid nodules have been published and vascular patterns of thyroid nodules were extensively studied (4). However, the question of placing the needle tip according to the vascular pattern is yet to be answered.

\section{Objectives}

This study was designed to assess whether the hypervascular or hypovascular site of a solid thyroid nodule would reveal more cellular aspirates when USFNA is performed under CDS guidance.

\section{Patients and Methods}

The study group consisted of patients with thyroid nodules referred to Bursa State Hospital radiology department for USFNA. In our institution, USFNA indications are based on previously published guidelines (2). Institutional review board approved the study protocol and informed consents were obtained from all patients enrolled in the study. Between January 2011 and June 2011, 23 consecutive patients (19 females, 4 males; mean age of 49.7, 28-71) referred for USFNA with solid nodules greater than $20 \mathrm{~mm}$ and clear distinction between hypervascular and hypovascular parts were included in the study prospectively. Cystic and degenerated/necrotic nodules were not included in the study population. All patients were examined with gray scale thyroid US followed by CDS by the same investigator (U.O.) with 10 years of relevant experience. The US unit uses Logic 400 Pro (GE Medical Systems, Kyunggi-Do, Korea) equipped with 6 - 9 MHz linear trans-

Copyright (C) 2015, Tehran University of Medical Sciences and Iranian Society of Radiology. This is an open-access article distributed under the terms of the Creative Commons Attribution-NonCommercial 4.0 International License (http://creativecommons.org/licenses/by-nc/4.0/) which permits copy and redistribute the material just in noncommercial usages, provided the original work is properly cited. 
ducer. The size of each nodule was recorded based on its largest diameter. For all nodules, with freehand technique and under direct CDS visualization, a 25-gauge needle attached to a 20-mL plastic syringe was placed in the targeted part; the syringe plunger was pulled back by $5 \mathrm{~mL}$. The first pass was from the hypovascular site. For hypovascular aspirate, the needle tip was located at least $10 \mathrm{~mm}$ to the nearest vessel and as central as possible to the nodule. The second pass was from the hypervascular site and the needle tip was placed in the most hypervascular part of the nodule as close as possible to the blood vessels whilst avoiding intravascular sampling. Single pass was used for each sample (hypo- and hypervascular) and the first pass was from the hypovascular site to avoid potential hemorrhage from adjacent vessels of the hypervascular site. When the needle was placed in the desired site, aspiration was made until material reached the hub and the needle tip was kept in place during aspiration without moving in the tissue to avoid any trauma or hemorrhage.

All samples examined by the same pathologist (S.A.) with 12 years of thyroid cytology experience blinded to patient data. The smears were air-dried, because MayGrünwald-Giemsa (MGG) stain was preferred for detailed analysis of cytoplasm and colloid. Smears were evaluated using the cytological features of smear cellularity, groups or single cells, background features and classified as follows; score 1: the number of cell groups less than 5: inadequate specimen, score 2: the number of thyrocyte groups between 5 to 10 , score 3: specimens with more than 10 cell groups. Endothelial cells were also noted as randomly scattered single endothelial cells $=1$ and groups of endothelial cells $=2$. The rate of adequate sampling between hypo- and hypervascular regions was calculated by McNemar test and the results were further statistically evaluated with weighted Kappa and power analysis.

\section{Results}

Twenty-three nodules (hypoechoic: 9, isoechoic: 2, hyperechoic: 4, heterogeneous and calcified: 8 ) in 23 patients (mean nodule diameter: $35.8 \mathrm{~mm}(20-100 \mathrm{~mm}$ ) were aspirated. Nineteen had benign cytology and 2 (9.5\%) had malignant cells indicative of papillary carcinoma. In two cases, the specimens did not contain adequate number of cells for pathologic assessment (Table 1). Ad- equate sampling was limited to $65 \%$ (15/23) of cases when only hypervascular site was used (Figure 1) and 83\% (19/23) of cases when only hypovascular site was used.

When both sites were evaluated together, overall adequate sampling was $91 \%$ (21/23). In hypovascular group $17 \%(4 / 23)$ of aspirates were inadequate compared to $35 \%$ (8/23) in hypervascular group. The adequate sampling rate between hypo- and hypervascular regions was not statistically significant according to McNemar test ( $\mathrm{P}$ $=0.289$ ) (Table 1). However, the calculated power of this data was 36\% (alpha error 0.05), which was low, limited by the number of patients. Adding a sample from hypervascular site to hypovascular aspirate increased the adequate sampling by $8 \%$. For pathological score, the weighted kappa for adequate sampling from hypovascular and hypervascular aspirates was calculated as 0,101 $\mathrm{P}$ $=0.473$. The pathological scores of hypo- and hypervascular site samples were in poor agreement. Thus, when the pathological score of one aspirate was high, the score of the same nodule tends to be low on the other aspirate and vice versa. As a result, when both sites were evaluated together, the number of nodules with cell groups more than 10 increased to 16 patients. The highest pathological score was achieved when both hypo- and hypervascular site aspirates were evaluated together (Table 2).

In hypovascular aspirates, groups of endothelial cells were observed in $17 \%(4 / 23)$ of the cases, whereas in hypervascular aspirates, groups of endothelial cells were observed in $52 \%(12 / 23)$ of the cases. The higher number of endothelial cell groups in hypervascular group did not affect the cytology scores.

\section{Discussion}

Thyroid nodule vascularity can be easily detected with CDS while performing USFNA, demonstrating the relative position of needle tip to the vessels. Our study showed that when the first aspirate is taken from the hypovascular site of a solitary nodule, adding a second pass from the hypervascular site would provide more cell groups for the pathological assessment. Therefore, the aspirates from the hypovascular and hypervascular sites of solid nodules are complementary and should be performed consecutively. In addition, increased number of endothelial cells in the hypervascular site aspirates does not decrease the cytology score of the nodule.

Table 1. Distribution of Adequacy of Cytologic Specimens Considering Both Hypovascular and Hypervascular Sites Aspirations

\begin{tabular}{lccc}
\hline Variables & \multicolumn{2}{c}{ Hypervascular Site Samples } & Total \\
\cline { 2 - 4 } & Inadequate & Adequate & \\
\hline Hypovascular Site Samples & 2 & 2 & 4 \\
Inadequate & 6 & 13 & 19 \\
Adequate & 8 & 15 & 23 \\
\hline Total & & & \\
\hline
\end{tabular}



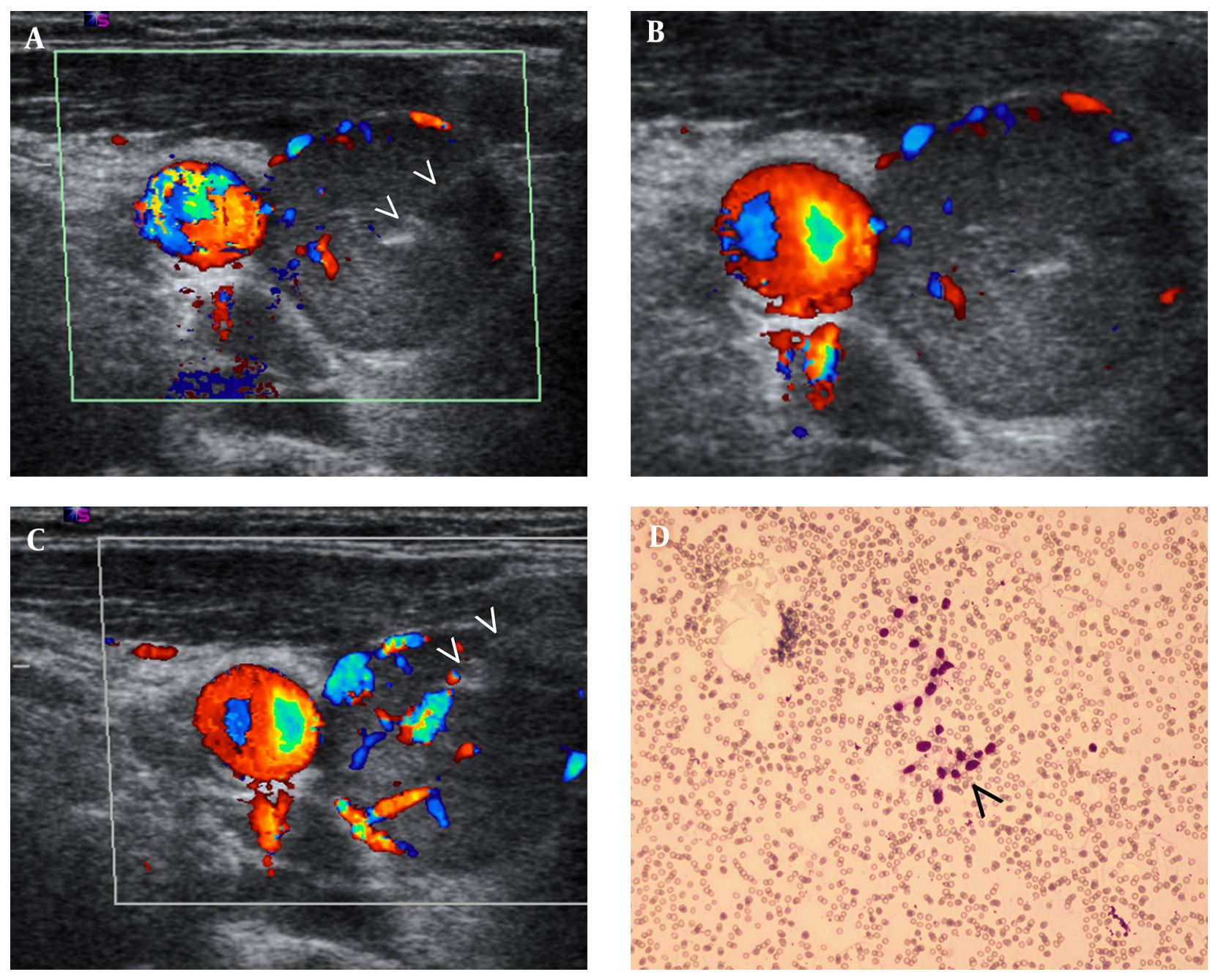

Figure 1. A sixty-five-year-old female with hypoechoic solid nodule. Aspiration from hypovascular site is shown with the needle tip at the center (arrows) (A) at different positions (B) or from hypervascular site (arrows) (C) Hypocellular sample from hypervascular site aspirate is shown. Thyrocytes are marked with arrowhead D. Final cytopathology diagnosis was hyperplastic nodule.

Table 2. Distribution of Cellularity Scores of Pathologic Assessments Considering Both Hypovascular and Hypervascular Sites Aspirations ${ }^{\text {a }}$

\begin{tabular}{lcccc}
\hline Pathology Score of Hypovascular & \multicolumn{4}{c}{ Pathology Score of Hypervascular Site Aspirate } \\
\cline { 2 - 5 } Site Aspirate & Score 1 $^{\text {b }}$ & Score 2 $^{\text {c }}$ & Score 3 $^{\text {d }}$ & Total \\
\hline Score 1 & 2 & 1 & 1 & 4 \\
Score 2 & 1 & 3 & 6 & 10 \\
Score 3 & 5 & 3 & 1 & 9 \\
Total & 8 & 7 & 8 & 23 \\
\hline
\end{tabular}

${ }^{\mathrm{a}}$ Weighted Kappa $=0.101, \mathrm{P}=0.473$.

b Score 1: The number of cell groups less than 5 , inadequate specimen.

c Score 2: The number of thyrocyte groups between 5 and 10 .

$\mathrm{d}$ Score 3: Specimens with more than 10 cell groups.

The biopsy guidelines recommend sampling the solid and viable parts of the tumor instead of cystic, necrotic or more sclerotic parts that may yield inadequate biopsy material. For example, in the diagnosis of bone tumors, a diligent search for more solid regions or areas of en- hancement at MR imaging or CT is considered vital in directing the biopsy to reach viable regions harboring diagnostic tissue (5). In patients with prostate disease, choosing the hypervascular site may preclude a positive result that yields carcinoma or inflammation (6). This 
raises the question of whether sampling from the hypervascular part of a thyroid nodule would yield better cytological results. Rausch et al. colleagues suggested the use of color Doppler "mapping" of the nodule immediately before needle aspiration to delineate perinodular flow and larger blood vessels within the nodule to avoid injury during the aspiration biopsy and reduce the amount of blood in the aspirate (1). In this study, nodules larger than $2 \mathrm{~cm}$ were included to demonstrate the hypo- and hypervascular sites separately. It has been reported that the lowest rate of specimen adequacy was observed among nodules larger than $3 \mathrm{~cm}$ due to increased vascularity and the larger size of blood vessels, with resultant bloodstaining of the material (3). In accordance with their findings, we observed that if the aspirate is solely from the hypervascular part of a large nodule, adequate sampling is limited to $65 \%$. However, intravascular sampling and blood staining may be avoided using CDS guidance. However, in nodules larger than $2 \mathrm{~cm}$, if the first aspirate is taken from hypovascular site of the nodule, preferring the hypervascular site for the second pass would yield $8 \%$ increase in adequate sampling. Preferring hypo- or hypervascular sites for aspiration did not yield statistically significant difference for adequate sampling. Thus, we suggest that, instead of avoiding hypervascular sites whilst sampling, CDS guidance should be used to avoid intravascular sampling since this approach may reveal better complementary results.

The overall incidence of cancer in patients with thyroid nodules selected for fine needle aspiration is approximately $9.2 \%-13.0$ (7). In this series, $9.5 \%$ of the nodules were malignant, probably because only large and solid nodules were investigated in the study. This is a limitation since there are various different US features proven suspicious for malignancy $(2,7)$. Larger series including smaller nodules as well as various nodules with US features suggestive of malignancy should be tested to verify the results of this study. In addition, a different study with additional techniques to take better samples like two consecutive passes from suspicious and/or vascular sites with control groups should be conducted. The sample size was also limited with insufficient power to dem- onstrate the superiority of one site to the other and the results of this study should be verified with larger series.

In conclusion, CDS may improve aspiration results by enabling determination of the appropriate site. The aspirates from hypovascular and hypervascular sites of solid nodules are complementary and should be performed consecutively for obtaining higher number of cell groups.

\section{Acknowledgements}

We thank associate professor Dr. Nadi Bakirci for his support in statistical analysis.

\section{Authors' Contributions}

Study concept and design, drafting of the manuscript and statistical analysis were conducted by Umit Aksoy Ozcan. Analysis and interpretation of data and critical revision of the manuscript for important intellectual content were performed by Umit Aksoy Ozcan and Safak Atahan.

\section{References}

1. Rausch P, Nowels K, Jeffrey RB, Jr. Ultrasonographically guided thyroid biopsy: a review with emphasis on technique. J Ultrasound Med. 2001;20(1):79-85.

2. Kim MJ, Kim EK, Park SI, Kim BM, Kwak JY, Kim SJ, et al. US-guided fine-needle aspiration of thyroid nodules: indications, techniques, results. Radiographics. 2008;28(7):1869-86.

3. Degirmenci B, Haktanir A, Albayrak R, Acar M, Sahin DA, Sahin O, et al. Sonographically guided fine-needle biopsy of thyroid nodules: the effects of nodule characteristics, sampling technique, and needle size on the adequacy of cytological material. Clin Radiol. 2007;62(8):798-803.

4. Moon HJ, Kwak JY, Kim MJ, Son EJ, Kim EK. Can vascularity at power Doppler US help predict thyroid malignancy? Radiology. 2010;255(1):260-9.

5. Jelinek JS, Murphey MD, Welker JA, Henshaw RM, Kransdorf MJ, Shmookler BM, et al. Diagnosis of primary bone tumors with image-guided percutaneous biopsy: experience with 110 tumors. Radiology. 2002;223(3):731-7.

6. Newman JS, Bree RL, Rubin JM. Prostate cancer: diagnosis with color Doppler sonography with histologic correlation of each biopsy site. Radiology.1995;195(1):86-90.

7. Frates MC, Benson CB, Charboneau JW, Cibas ES, Clark OH, Coleman BG, et al. Management of thyroid nodules detected at US: Society of Radiologists in Ultrasound consensus conference statement. Radiology. 2005;237(3):794-800. 